\title{
New Explicit Lorentzian Einstein-Weyl Structures in 3-Dimensions
}

Joël MERKER † and Pawet NUROWSKI

† Laboratoire de Mathématiques d'Orsay, Université Paris-Sud, CNRS, Université Paris-Saclay, 91405 Orsay Cedex, France

E-mail: joel.merker@universite-paris-saclay.fr

URL: http://www.imo.universite-paris-saclay.fr/ merker/

$\ddagger$ Centrum Fizyki Teoretycznej, Polska Akademia Nauk,

Al. Lotników 32/46, 02-668 Warszawa, Poland

E-mail: nurowski@cft.edu.pl

URL: http://www.fuw.edu.pl/ nurowski/

Received March 30, 2020, in final form June 08, 2020; Published online June 17, 2020

https://doi.org/10.3842/SIGMA.2020.056

Abstract. On a 3D manifold, a Weyl geometry consists of pairs $(g, A)=$ (metric, 1 -form) modulo gauge $\widehat{g}=\mathrm{e}^{2 \varphi} g, \widehat{A}=A+\mathrm{d} \varphi$. In 1943, Cartan showed that every solution to the Einstein-Weyl equations $R_{(\mu \nu)}-\frac{1}{3} R g_{\mu \nu}=0$ comes from an appropriate 3D leaf space quotient of a 7D connection bundle associated with a $3^{\text {rd }}$ order ODE $y^{\prime \prime \prime}=H\left(x, y, y^{\prime}, y^{\prime \prime}\right)$ modulo point transformations, provided 2 among 3 primary point invariants vanish

Wünschmann $(H) \equiv 0 \equiv \operatorname{Cartan}(H)$.

We find that point equivalence of a single $\mathrm{PDE} z_{y}=F\left(x, y, z, z_{x}\right)$ with para-CR integrability $D F:=F_{x}+z_{x} F_{z} \equiv 0$ leads to a completely similar $7 \mathrm{D}$ Cartan bundle and connection. Then magically, the (complicated) equation Wünschmann $(H) \equiv 0$ becomes

$$
0 \equiv \text { Monge }(F):=9 F_{p p}^{2} F_{p p p p p}-45 F_{p p} F_{p p p} F_{p p p p}+40 F_{p p p}^{3}, \quad p:=z_{x},
$$

whose solutions are just conics in the $\{p, F\}$-plane. As an ansatz, we take

$$
F(x, y, z, p):=\frac{\alpha(y)(z-x p)^{2}+\beta(y)(z-x p) p+\gamma(y)(z-x p)+\delta(y) p^{2}+\varepsilon(y) p+\zeta(y)}{\lambda(y)(z-x p)+\mu(y) p+\nu(y)},
$$

with 9 arbitrary functions $\alpha, \ldots, \nu$ of $y$. This $F$ satisfies $D F \equiv 0 \equiv \operatorname{Monge}(F)$, and we show that the condition $\operatorname{Cartan}(H) \equiv 0$ passes to a certain $K(F) \equiv 0$ which holds for any choice of $\alpha(y), \ldots, \nu(y)$. Descending to the leaf space quotient, we gain $\infty$-dimensional functionally parametrized and explicit families of Einstein-Weyl structures $[(g, A)]$ in 3D. These structures are nontrivial in the sense that $\mathrm{d} A \not \equiv 0$ and $\operatorname{Cotton}([g]) \not \equiv 0$.

Key words: Einstein-Weyl structures; Lorentzian metrics; para-CR structures; third-order ordinary differential equations; Monge invariant; Wünschmann invariant; Cartan's method of equivalence; exterior differential systems

2020 Mathematics Subject Classification: 83C15; 53C25; 83C20; 53C25; 53C10; 53C25; 53A30; 53A 55; 34A26; 34C14; 58A $15 ; 53-08$

\section{Introduction}

On an $n$-manifold $M$, a Weyl geometry is a pair $(g, A)$ of a signature $(k, n-k)$ pseudo-Riemannian metric modulo $\widehat{g}=\mathrm{e}^{2 \varphi} g$ together with a 1 -form $A$ modulo $\widehat{A}=A+\mathrm{d} \varphi$, where $\varphi: M \rightarrow \mathbb{R}$ is any function. As in Riemannian geometry, a symmetric Ricci tensor $R_{(\mu \nu)}$ with scalar curvature $R$ 
can be defined (see $[3,7,8]$ or Section 2). The Einstein-Weyl equations in vacuum

$$
R_{(\mu \nu)}-\frac{1}{n} R g_{\mu \nu}=0, \quad 1 \leqslant \mu, \nu \leqslant n,
$$

which depend only on the class $[(g, A)]$, have raised interest, specially in dimension $n=3$. We find various functionally parametrized explicit families of solutions. On $\mathbb{R}^{3} \ni(x, y, z)$, take for instance 5 free arbitrary functions $b, c, k, l, m$ of $y$ with derivatives $b^{\prime}, k^{\prime}$.

Theorem 1.1. All pairs $(g, A)$ such that

$$
\begin{aligned}
g:= & (k+b z)^{2} \mathrm{~d} x^{2}+x^{2}\left(l^{2}-c m\right) \mathrm{d} y^{2}+x^{2} b^{2} \mathrm{~d} z^{2} \\
& +2 x(c k z-b l z+k l-b m) \mathrm{d} x \mathrm{~d} y-2 x b(k+b z) \mathrm{d} x \mathrm{~d} z-2 x^{2}(c k-b l) \mathrm{d} y \mathrm{~d} z, \\
A:= & \frac{-c k+b l+b^{\prime} k-b k^{\prime}}{x\left(c k^{2}-2 b k l+b^{2} m\right)}(x b \mathrm{~d} z-(k+b z) \mathrm{d} x) \\
& +\frac{b l^{2}-c b m-b^{\prime} k l+b b^{\prime} m+c k k^{\prime}-b k^{\prime} l}{c k^{2}-2 b k l+b^{2} m} \mathrm{~d} y,
\end{aligned}
$$

satisfy equations (1.1), hence define a Lorentzian Einstein-Weyl structure on $\mathbb{R}^{3}$.

Moreover, all such examples are generically conformally non-flat, and each of the 5 independent components of the Cotton tensor of the underlying conformal structure $(M,[g])$ is not identically zero.

We discover in fact even more general explicit families of solutions depending on 9 free arbitrary functions of 1 variable $y$. Explicit examples of Einstein-Weyl structures in 3D were known before $[1,3,4,5,7,8,10,11,12,17,18,19,20]$.

According to [3], all Einstein-Weyl structures may be constructed by a certain quotient process from a 7D Cartan bundle associated with equivalences of $3^{\text {rd }}$ order ordinary differential equations. Those, in turn, are known to be para-CR structures of type $(1,1,2)$, cf. [9, Section 5.1.3].

In the present paper, we explore the observation that PDEs on the plane $(x, y)$ of the form $z_{y}=F\left(x, y, z, z_{x}\right)$, considered modulo point transformations, also happen to be $(1,1,2)$ para-CR structures, in certain circumstances. In Section 8, we show how equivalence classes of $(1,1,2)$ para-CR structures associated to PDEs $z_{y}=F$ are 'embedded' into the space of equivalence classes of $3^{\text {rd }}$ order ODEs. This distinguishes a certain class of $3^{\text {rd }}$ order ODEs from which we construct our explicit solutions to the Einstein-Weyl equations.

Thus, our main approach is to study point equivalences of a single PDE of the form (novelty)

$$
z_{y}=F\left(x, y, z, z_{x}\right),
$$

with unknown $z=z(x, y)$. From para-CR geometry [9, 13], an integrability condition is required, namely,

$$
D F:=F_{x}+z_{x} F_{z} \equiv 0 .
$$

To exclude trivial PDEs, another point invariant condition must be assumed:

$$
F_{p p} \neq 0 \quad\left(\text { abbreviate } p:=z_{x}\right) .
$$

In Theorem 5.2, we construct a 7-dimensional Cartan bundle/connection $P_{7} \longrightarrow J_{4} \ni$ $(x, y, z, p)$ canonically associated to point equivalences of such PDEs $z_{y}=F\left(x, y, z, z_{x}\right)$, we determine a canonical coframe $\left\{\theta^{1}, \theta^{2}, \theta^{3}, \theta^{4}, \Omega_{1}, \Omega_{2}, \Omega_{3}\right\}$ on $P_{7}$, and we find that its structure equations (4.4) incorporate exactly 3 primary invariants, named $A_{1}, B_{1}, C_{1}$. 
Quite unexpectedly, we realize that these structure equations have the same form as the structure equations of the canonical 7-dimensional Cartan bundle/connection associated with point equivalences of $3^{\text {rd }}$ order ODEs $y^{\prime \prime \prime}=H\left(x, y, y^{\prime}, y^{\prime \prime}\right)$. Furthermore, it is known that quite similarly, 3 primary differential invariants govern such geometries. Two among them are: the Wünschmann invariant $W(H)$ [22] and the Cartan invariant $C(H)$ [2, 3]. Since Cartan 1943, it is also known $[3,6,7,8,10]$ that all solutions to the Einstein-Weyl structure equations (1.1) can be obtained from ODEs satisfying $W(H) \equiv 0 \equiv C(H)$. Translating what is known for ODEs or performing computations from scratch, we will set up and state Cartan's construction from the PDE side, see Theorem 5.3.

But from the ODE side unfortunately, it is quite difficult to solve Wünschmann's nonlinear equation incorporating 25 differential monomials

$$
\begin{aligned}
0 \equiv W(H):= & -18 q H_{q} H_{p q}+9 p H_{y} H_{q q}+18 q H H_{p q q}+9 q H_{p} H_{q q}-18 p H_{q} H_{y q}+18 p H H_{y q q} \\
& -9 H H_{q} H_{q q}+18 p q H_{y p q}+18 p H_{x y q}+18 q H_{x p q}+9 H_{x} H_{q q}+18 H H_{x q q} \\
& -18 H_{q} H_{x q}+18 H_{p} H_{q}+9 H_{x x q}-27 H_{x p}+4 H_{q}^{3}+9 p^{2} H_{y y q}-27 p H_{y p} \\
& +9 q H_{y q}+9 q^{2} H_{p p q}-27 q H_{p p}-18 H H_{p q}+9 H^{2} H_{q q q}+54 H_{y} .
\end{aligned}
$$

This inspired us to try to work on the PDE side $z_{y}=F\left(x, y, z, z_{x}\right)$, instead of the ODE side. Then magically, $W(H) \equiv 0$ transforms into the much simpler classical invariant of Monge [16]

$$
0 \equiv \operatorname{Monge}(F):=9 F_{p p}^{2} F_{p p p p p}-45 F_{p p} F_{p p p} F_{p p p p}+40 F_{p p p}^{3},
$$

When $F_{p p} \neq 0$, it is known that $M(F) \equiv 0$ holds if and only if there exist functions A, B, C, K, $\mathrm{L}, \mathrm{M}$ of $(x, y, z)$ such that

$$
0 \equiv \mathrm{A} F^{2}+2 \mathrm{~B} F p+\mathrm{C} p^{2}+2 \mathrm{~K} F+2 \mathrm{~L} p+\mathrm{M} \text {. }
$$

Assuming $\mathrm{A}:=0$, we obtain the following

Proposition 1.2. The general solution $F=F(x, y, z, p)$ to

$$
\begin{aligned}
& 0 \equiv F_{x}+p F_{z}, \\
& 0 \equiv 0+2 \mathrm{~B} F p+\mathrm{C} p^{2}+2 \mathrm{~K} F+2 \mathrm{~L} p+\mathrm{M}
\end{aligned}
$$

is

$$
F=\frac{\alpha(y)(z-x p)^{2}+\beta(y)(z-x p) p+\gamma(y)(z-x p)+\delta(y) p^{2}+\varepsilon(y) p+\zeta(y)}{\lambda(y)(z-x p)+\mu(y) p+\nu(y)},
$$

with 9 arbitrary functions $\alpha, \beta, \gamma, \delta, \varepsilon, \zeta, \lambda, \mu, \nu$ of $y$.

Of course, to the Cartan invariant $C(H)$ from the ODE side there corresponds from the PDE side a certain invariant we name $K(F)$ : its expression appears in Theorem 5.2. Miraculously, then, a direct calculation shows that no further constraint is imposed.

Proposition 1.3. For any choice of $\alpha(y), \beta(y), \gamma(y), \delta(y), \varepsilon(y), \zeta(y), \lambda(y), \mu(y), \nu(y)$, the second condition

$$
K\left(F_{\alpha, \ldots, \nu}\right) \equiv 0
$$

for obtaining Weyl pairs $[(g, A)]$ satisfying the Einstein-Weyl field equations (1.1) holds automatically. 
We then get - quite long - formulas for pairs $[(g, A)]$ expressed explicitly in terms of $\alpha, \beta, \gamma$, $\delta, \varepsilon, \zeta, \lambda, \mu, \nu$. The subfamily for which $\beta=0, \delta=0, \varepsilon=0, \mu=0$ corresponds (with different notations) to Theorem 1.1.

Theorem 1.4. Same conclusion as in Theorem 1.1 with

$$
\begin{aligned}
g & :=\tau^{1} \tau^{2}+\tau^{2} \tau^{1}+\tau^{3} \tau^{3}, \\
A:=\tau^{3} \frac{1}{2 \Pi}( & \gamma \lambda x-\gamma \mu+x \lambda \nu^{\prime}+\beta \lambda z+\lambda \mu^{\prime} z-2 \alpha \mu z \\
& \left.\quad-\lambda^{\prime} \mu z-\mu \nu^{\prime}-x \lambda^{\prime} \nu-2 x \alpha \nu+\beta \nu+\mu^{\prime} \nu\right),
\end{aligned}
$$

with the coframe

$$
\begin{aligned}
\tau^{1}:= & \mathrm{d} x+\frac{\mathrm{d} y}{x \lambda-\mu}\left(x \beta-\delta-x^{2} \alpha\right), \\
\tau^{2}:= & \frac{2 \mathrm{~d} y}{x \lambda-\mu} \Pi, \\
\tau^{3}:= & (-\lambda z-\nu) \mathrm{d} x+\frac{1}{x \lambda-\mu} \mathrm{d} y\left(-\varepsilon \mu+2 x^{2} \alpha \nu+x \gamma \mu-2 x \beta \nu-\beta \mu z+2 \delta \lambda z+2 x \alpha \mu z\right. \\
& \left.+x \varepsilon \lambda+2 \delta \nu-x^{2} \gamma \lambda-x \beta \lambda z\right)+(x \lambda-\mu) \mathrm{d} z,
\end{aligned}
$$

and the function

$$
\begin{aligned}
\Pi:= & x^{2} \zeta \lambda^{2}+\alpha \mu^{2} z^{2}+2 x \alpha \mu \nu z+x^{2} \alpha \nu^{2}-\beta \lambda \mu z^{2}-x \beta \lambda \nu z+\delta \lambda^{2} z^{2}+x \varepsilon \lambda^{2} z-2 x \zeta \lambda \mu \\
& -\beta \mu \nu z-x \beta \nu^{2}+2 \delta \lambda \nu z-\varepsilon \lambda \mu z+x \varepsilon \lambda \nu-x \gamma \lambda \mu z-x^{2} \gamma \lambda \nu+\zeta \mu^{2}+\delta \nu^{2}-\varepsilon \mu \nu \\
& +\gamma \mu^{2} z+x \gamma \mu \nu,
\end{aligned}
$$

again with $\mathrm{d} A \not \equiv 0$ and $\operatorname{Cotton}([g]) \not \equiv 0$.

At the end, we also present other families of functionally parametrized solutions, when $\mathrm{A} \neq 0$.

\section{Weyl geometry: a summary}

In Einstein's theory, gravity is described in terms of a (pseudo-)riemannian metric $g$ called the gravitational potential. In Maxwell's theory, the electromagnetic field is described in terms of a 1 -form $A$ called the Maxwell potential.

In his attempt Raum, Zeit, Materie [21] of unifying gravitation and electromagnetism, Weyl was inspired to introduce the synthetic geometric structure on any $n$-dimensional manifold $M^{n}$ which consists of classes of such pairs $[(g, A)]$ under the equivalence relation

$$
(g, A) \sim(\widehat{g}, \widehat{A})
$$

holding by definition if and only if there exists a function $\varphi: M \longrightarrow \mathbb{R}$ such that

(1) $\widehat{g}=\mathrm{e}^{2 \varphi} g$;

(2) $\widehat{A}=A+\mathrm{d} \varphi$.

Clearly, the electromagnetic field strength $F:=\mathrm{d} A$ depends only on the class. The signature $(k, n-k)$ of $g$ can be arbitrary. Conformally Einstein structures from ordinary conformal geometry are a special class of Weyl structures, corresponding to the choice of a closed - hence locally exact - 1-form $A$.

Inspired by Levi-Civita, Weyl established that to such a Weyl structure $(M,[(g, A)])$ is associated a unique connection $D$ on $T M$ satisfying: 
(A) D has no torsion;

(B) $D g=2 A g$ for any representative $(g, A)$ of the class $[(g, A)]$.

In any (local) coframe $\omega^{\mu}, \mu=1, \ldots, n$, for the cotangent bundle $T^{*} M$ in which $g=g_{\mu \nu} \omega^{\mu} \omega^{\nu}$, the connection 1-forms $\Gamma^{\mu}{ }_{\nu}$ of $D$, or equivalently the $\Gamma_{\mu \nu}:=g_{\mu \rho} \Gamma^{\rho}{ }_{\nu}$, are indeed uniquely defined from the more explicit conditions:

$\left(\mathrm{A}^{\prime}\right) \mathrm{d} \omega^{\mu}+\Gamma_{\nu}^{\mu} \wedge \omega^{\nu}=0$

$\left(\mathrm{B}^{\prime}\right) D g_{\mu \nu}:=\mathrm{d} g_{\mu \nu}-\Gamma_{\mu \nu}-\Gamma_{\nu \mu}=2 A g_{\mu \nu}$.

Then the curvature of this Weyl connection identifies with the collection of $n^{2}$ curvature 2 -forms

$$
{\Omega^{\mu}}_{\nu}:=\mathrm{d} \Gamma^{\mu}{ }_{\nu}+\Gamma_{\rho}^{\mu} \wedge \Gamma_{\nu}^{\rho}
$$

which produce the curvature tensor $R^{\mu}{ }_{\nu \rho \sigma}$ by expanding in the given coframe $\omega^{\mu}$

$$
{\Omega^{\mu}}_{\nu}=\frac{1}{2} R_{\nu \rho \sigma}^{\mu} \omega^{\rho} \wedge \omega^{\sigma} .
$$

It turns out that $R_{\nu \rho \sigma}^{\mu}$ is a tensor density, which means in particular that its vanishing is independent of the choice of a representative $(g, A)$, and hence as such, serves as a starting point for all invariants of a Weyl geometry $(M,[(g, A)])$, produced by covariant differentiation.

Other invariant objects are:

- the (Weyl-)Ricci tensor $R_{\mu \nu}:=R_{\mu \rho \nu}^{\rho}$;

- its symmetric part $R_{(\mu \nu)}:=\frac{1}{2}\left(R_{\mu \nu}+R_{\nu \mu}\right)$;

- its antisymmetric part $R_{[\mu \nu]}:=\frac{1}{2}\left(R_{\mu \nu}-R_{\nu \mu}\right)$.

In particular, an appropriately contracted Bianchi identity shows that in 3-dimensions

$$
R_{[\mu \nu]}=-\frac{3}{2} F_{\mu \nu},
$$

where $F=\mathrm{d} A=: \frac{1}{2} F_{\mu \nu} \omega^{\mu} \wedge \omega^{\nu}$.

In [3], Élie Cartan proposed dynamical Einstein equations for a Weyl geometry $(M,[(g, A)])$ postulating that the trace-free part of the symmetric Ricci tensor vanishes

$$
R_{(\mu \nu)}-\frac{1}{n} R g_{\mu \nu}=0
$$

where $R:=g^{\mu \nu} R_{\mu \nu}$, with $g^{\mu \rho} g_{\rho \nu}=\delta^{\mu}{ }_{\nu}$ and $n=\operatorname{dim} M$.

These equations (2.1) are called Einstein-Weyl equations, and a Weyl geometry satisfying (2.1) is called an Einstein-Weyl structure. The reason for this name is as follows.

Since a Weyl structure $(M,[g, A])$ with vanishing $F=\mathrm{d} A \equiv 0$ is equivalent to a plain (pseudo-)conformal structure $(M,[g])$ and since the Weyl connection $D$ then reduces to the LeviCivita connection, these equations (2.1) are a natural generalization of Einstein's field equations. According to Weyl's approach, a gravity potential $g$ is thereby coupled with an electromagnetic field $F=\mathrm{d} A$. 


\section{Cartan's solution to the Einstein-Weyl vacuum equations}

In [2], Cartan gave a geometric description of all solutions to the Einstein-Weyl equations (2.1) in 3-dimensions. In particular, he showed that there is a one-to-one correspondence between $3^{\text {rd }}$-order ODEs $y^{\prime \prime \prime}=H\left(x, y, y^{\prime}, y^{\prime \prime}\right)$ considered modulo point transformations of variables which satisfy certain two point-invariant conditions

$$
\begin{aligned}
& W(H) \equiv 0, \\
& C(H) \equiv 0,
\end{aligned}
$$

and 3-dimensional Einstein-Weyl structures with Lorentzian metrics $g$ of signature $(2,1)$. Abbreviating $p:=y^{\prime}, q:=y^{\prime \prime}$, in terms of the total differentiation operator

$$
D:=\partial_{x}+p \partial_{y}+q \partial_{p}+H \partial_{q}
$$

their explicit expressions are

$$
\begin{aligned}
& W:=9 D D H_{q}-27 D H_{p}-18 H_{q} D H_{q}+18 H_{q} H_{p}+4 H_{q}^{3}+54 H_{y}, \\
& C:=18 H_{q q} D H_{q}-12 H_{q q} H_{q}^{2}-54 H_{q q} H_{p}+36 H_{p q} H_{q}-108 H_{y q}+54 H_{p p} .
\end{aligned}
$$

Although Cartan's geometric arguments [3] offer, in the Lorentzian setting, a complete - but abstract - understanding of the space of all solutions of the Einstein-Weyl equations (2.1), it is quite difficult to find explicit solutions to the Wünschmann-Cartan equations $0 \equiv W(H) \equiv C(H)$, which would provide workable formulas for such Einstein-Weyl structures.

Some particular solutions are known, e.g.,

$$
H=\frac{3 q^{2}}{2 p}, \quad H=\frac{3 q^{2} p}{p^{2}+1}, \quad H=q^{3 / 2}, \quad H=\alpha \frac{\left(2 q y-p^{2}\right)^{3 / 2}}{y^{2}}, \quad \alpha \in \mathbb{R},
$$

or the 'horrible'

$$
H=\frac{p q(-12+3 p q-8 \sqrt{1-p q})+8(1+\sqrt{1-p q})}{p^{3}} .
$$

They were all obtained by rather ad hoc methods.

In fact, the main difficulty in getting a systematic approach to finding the solutions is an annoying nonlinearity of the Wünschmann condition $W \equiv 0$.

\section{Third-order ODEs modulo point transformations of variables}

It was Cartan [2] who solved the equivalence problem for $3^{\text {rd }}$ order ODEs considered modulo point transformations. Nowadays, the result may be stated more elegantly in terms of a certain Cartan connection $[7,8]$, as follows.

To any $3^{\text {rd }}$ order ODE

$$
y^{\prime \prime \prime}=H\left(x, y, y^{\prime}, y^{\prime \prime}\right),
$$

one associates a contact-like coframe on the space $J_{4} \ni(x, y, p, q)$ of 2-jets of graphs $x \longmapsto y(x)$ :

$$
\omega^{1}:=\mathrm{d} y-p \mathrm{~d} x, \quad \omega^{2}:=\mathrm{d} x, \quad \omega^{3}:=\mathrm{d} p-q \mathrm{~d} x, \quad \omega^{4}:=\mathrm{d} q-H(x, y, p, q) \mathrm{d} x .
$$

It follows that if a $3^{\text {rd }}$ order ODE (4.1) undergoes a point transformation of variables

$$
(x, y) \longmapsto(\bar{x}, \bar{y})=(\bar{x}(x, y), \bar{y}(x, y)),
$$


then the 1-forms $\left(\omega^{1}, \omega^{2}, \omega^{3}, \omega^{4}\right)$ transform as

$$
\left(\begin{array}{l}
\omega^{1} \\
\omega^{2} \\
\omega^{3} \\
\omega^{4}
\end{array}\right) \longmapsto\left(\begin{array}{cccc}
\mathbf{u}_{1} & 0 & 0 & 0 \\
\mathbf{u}_{2} & \mathbf{u}_{3} & 0 & 0 \\
\mathbf{u}_{4} & 0 & \mathbf{u}_{5} & 0 \\
\mathbf{u}_{6} & 0 & \mathbf{u}_{7} & \mathbf{u}_{8}
\end{array}\right)\left(\begin{array}{c}
\omega^{1} \\
\omega^{2} \\
\omega^{3} \\
\omega^{4}
\end{array}\right)=:\left(\begin{array}{c}
\theta^{1} \\
\theta^{2} \\
\theta^{3} \\
\theta^{4}
\end{array}\right),
$$

where the $\mathrm{u}_{i}$ are certain functions on $J_{4}$.

Actually, Cartan assures us that the entire equivalence problem for $3^{\text {rd }}$ order ODEs considered modulo point transformations of variables is the same as the equivalence problem for 1 -forms (4.2), considered modulo transformations (4.3). There is a unique way of reducing these eight group parameters $\mathrm{u}_{i}$ to only three $\mathrm{u}_{3}, \mathrm{u}_{5}, \mathrm{u}_{7}$, the other ones being expressed in terms of them. This is achieved by forcing the exterior differentials of the $\theta^{\mu}$ 's to satisfy the EDS (4.4) below.

Theorem 4.1 ([2, 7, 8]). A $3^{\text {rd }}$ order ODE $y^{\prime \prime \prime}=H\left(x, y, y^{\prime}, y^{\prime \prime}\right)$ with its associated 1-forms

$$
\omega^{1}=\mathrm{d} y-p \mathrm{~d} x, \quad \omega^{2}=\mathrm{d} x, \quad \omega^{3}=\mathrm{d} p-q \mathrm{~d} x, \quad \omega^{4}=\mathrm{d} q-H(x, y, p, q) \mathrm{d} x,
$$

uniquely defines a 7-dimensional fiber bundle $P_{7} \longrightarrow J_{4}$ over the space of second jets $J_{4} \ni$ $(x, y, p, q)$ and a unique coframe $\left\{\theta^{1}, \theta^{2}, \theta^{3}, \theta^{4}, \Omega_{1}, \Omega_{2}, \Omega_{3}\right\}$ on $P_{7}$ enjoying structure equations of the shape

$$
\begin{aligned}
\mathrm{d} \theta^{1}= & \Omega_{1} \wedge \theta^{1}-\theta^{2} \wedge \theta^{3}, \\
\mathrm{~d} \theta^{2}= & \left(\Omega_{1}-\Omega_{3}\right) \wedge \theta^{2}+\boldsymbol{B}_{1} \theta^{1} \wedge \theta^{3}-\boldsymbol{B}_{2} \theta^{1} \wedge \theta^{4}, \\
\mathrm{~d} \theta^{3}= & \Omega_{2} \wedge \theta^{1}+\Omega_{3} \wedge \theta^{3}+\theta^{2} \wedge \theta^{4}, \\
\mathrm{~d} \theta^{4}= & \left(2 \Omega_{3}-\Omega_{1}\right) \wedge \theta^{4}-\Omega_{2} \wedge \theta^{3}-\boldsymbol{A}_{1} \theta^{1} \wedge \theta^{2}, \\
\mathrm{~d} \Omega_{1}= & \Omega_{2} \wedge \theta^{2}+\left(\boldsymbol{A}_{2}-2 \boldsymbol{C}_{1}\right) \theta^{1} \wedge \theta^{2}+\left(3 \boldsymbol{B}_{3}+\boldsymbol{E}_{1}\right) \theta^{1} \wedge \theta^{3}+\left(2 \boldsymbol{B}_{1}-3 \boldsymbol{B}_{4}\right) \theta^{1} \wedge \theta^{4}+\boldsymbol{B}_{2} \theta^{3} \wedge \theta^{4}, \\
\mathrm{~d} \Omega_{2}= & \Omega_{2} \wedge\left(\Omega_{1}-\Omega_{3}\right)-\boldsymbol{A}_{3} \theta^{1} \wedge \theta^{2}+\boldsymbol{E}_{2} \theta^{1} \wedge \theta^{3}-\left(\boldsymbol{B}_{3}+\boldsymbol{E}_{1}\right) \theta^{1} \wedge \theta^{4}+\boldsymbol{C}_{1} \theta^{2} \wedge \theta^{3} \\
& +\left(\boldsymbol{B}_{1}-2 \boldsymbol{B}_{4}\right) \theta^{3} \wedge \theta^{4}, \\
\mathrm{~d} \Omega_{3}= & -\boldsymbol{C}_{1} \theta^{1} \wedge \theta^{2}+\left(2 \boldsymbol{B}_{3}+\boldsymbol{E}_{1}\right) \theta^{1} \wedge \theta^{3}+2\left(\boldsymbol{B}_{1}-\boldsymbol{B}_{4}\right) \theta^{1} \wedge \theta^{4}+2 \boldsymbol{B}_{2} \theta^{3} \wedge \theta^{4} .
\end{aligned}
$$

Moreover, two equations $y^{\prime \prime \prime}=H\left(x, y, y^{\prime}, y^{\prime \prime}\right)$ and $\bar{y}^{\prime \prime \prime}=\bar{H}\left(\bar{x}, \bar{y}, \bar{y}^{\prime}, \bar{y}^{\prime}\right)$ are locally point equivalent if and only if there exists a local bundle isomorphism $\Phi: P_{7} \stackrel{\sim}{\longrightarrow} \bar{P}_{7}$ between the corresponding bundles $P_{7} \longrightarrow J_{4}$ and $\bar{P}_{7} \longrightarrow \bar{J}_{4}$ satisfying

$$
\Phi^{*} \bar{\theta}^{\mu}=\theta^{\mu} \quad \text { and } \quad \Phi^{*} \bar{\Omega}_{i}=\Omega_{i}, \quad \mu=1,2,3,4, \quad i=1,2,3 .
$$

Exactly 3 (boxed) invariants are primary: $A_{1}, B_{1}, C_{1}$, while others express in terms of them and their covariant derivatives. Point equivalence to $\bar{y}^{\prime \prime \prime}=0$ is characterized by $0 \equiv A_{1} \equiv B_{1} \equiv$ $C_{1}$. Two relevant explicit expressions are

$$
\begin{aligned}
& A_{1}=\frac{1}{54} \frac{\mathrm{u}_{3}^{3}}{\mathrm{u}_{1}^{3}} W, \\
& C_{1}=\frac{1}{54} \frac{\mathrm{u}_{3}}{\mathrm{u}_{1}^{2}}\left(C+\frac{1}{27} W_{q}\right) .
\end{aligned}
$$

The seven 1 -forms $\left(\theta^{1}, \theta^{2}, \theta^{3}, \theta^{4}, \Omega_{1}, \Omega_{2}, \Omega_{3}\right)$ set up a Cartan connection $\widehat{\omega}$ on $P_{7}$ via

$$
\widehat{\omega}:=\left(\begin{array}{cccc}
\frac{1}{2} \Omega_{1} & \frac{1}{2} \Omega_{2} & 0 & 0 \\
-\theta^{2} & \Omega_{3}-\frac{1}{2} \Omega_{1} & 0 & 0 \\
\theta^{3} & -\theta^{4} & \frac{1}{2} \Omega_{1}-\Omega_{3} & -\frac{1}{2} \Omega_{2} \\
2 \theta^{1} & \theta^{3} & \theta^{2} & -\frac{1}{2} \Omega_{1}
\end{array}\right),
$$


and the structure equations (4.4) are just the equations for the curvature $\widehat{K}$ of this connection

$$
\mathrm{d} \widehat{\omega}+\widehat{\omega} \wedge \widehat{\omega}=: \widehat{K} .
$$

Now, the structure equations (4.4) guarantee that the bundle $P_{7}$ is foliated by a 4-dimensional distribution annihilating the three 1-forms $\left(\theta^{1}, \theta^{3}, \theta^{4}\right)$, and that the leaf space $M_{3}$ of this foliation is equipped with a natural Weyl geometry, if and only if two among three primary invariants vanish identically

$$
0 \equiv A_{1}(H) \equiv C_{1}(H)
$$

A representative $(g, A)$ of the concerned Weyl class $[(g, A)]$ on $M_{3}$ has then the signature $(2,1)$ symmetric bilinear form

$$
g:=\theta^{3} \theta^{3}+\theta^{1} \theta^{4}+\theta^{4} \theta^{1},
$$

which is obtained as the determinant of the lower-left $2 \times 2$ submatrix of the connection matrix $\widehat{\omega}$, while the 1-form is defined as

$$
A:=\Omega_{3}
$$

It is thanks to the hypothesis $A_{1} \equiv 0 \equiv C_{1}$ that $g$ and $A$, originally defined on $P_{7}$, descend on $M_{3}$.

Furthermore, according to a result of Cartan in [3], any such Weyl geometry $[(g, A)]$ defined on such a leaf space $M_{3}$ is automatically Einstein-Weyl!

We stress that given $H=H(x, y, p, q)$ satisfying $A_{1} \equiv 0 \equiv C_{1}$, or equivalently

$$
W(H) \equiv 0 \equiv C(H)
$$

one can in principle set up explicit formulas for the corresponding forms $\theta^{1}, \theta^{3}, \theta^{4}, \Omega_{3}$ on $P_{7}$, and this in turn can provide explicit formulas for $(g, A)$ on $M_{3}$. However, one substantial obstacle is

Question 4.2. How to solve $W(H) \equiv 0 \equiv C(H)$ ?

\section{$5 \quad$ PDE on the plane $z_{y}=F\left(x, y, z, z_{x}\right)$ modulo point transformations}

We recall that in [9], it was shown that the equivalence problem for $3^{\text {rd }}$-order ODEs considered modulo point transformations of variables is in one-to-one correspondence with the equivalence problem for 4-dimensional para-CR structures of type $(1,1,2)$, cf. also [14, 15]. This thus suggests a new approach for constructing Lorentzian Einstein-Weyl structures via para-CR structures of type $(1,1,2)$. Instead of working with general para-CR structures of type $(1,1,2)$, we will concentrate on a subclass determined in the following way.

We start with a class of PDEs of the form

$$
z_{y}=F\left(x, y, z, z_{x}\right),
$$

considered modulo point transformations, for an unknown function $z=z(x, y)$. We then ask when this class defines a para-CR structure of type $(1,1,2)$.

To answer this (in Proposition 5.1), we need a little preparation. Using the abbreviation $z_{x}=: p$, we indeed consider such PDEs modulo point transformations of variables

$$
(x, y, z) \longmapsto(\bar{x}, \bar{y}, \bar{z})=(\bar{x}(x, y, z), \bar{y}(x, y, z), \bar{z}(x, y, z)) .
$$


This leads to an equivalence problem for the four 1-forms

$$
\omega_{0}^{1}:=\mathrm{d} z-p \mathrm{~d} x-F(x, y, z, p) \mathrm{d} y, \quad \omega_{0}^{2}:=\mathrm{d} p, \quad \omega_{0}^{3}:=\mathrm{d} x, \quad \omega_{0}^{4}:=\mathrm{d} y,
$$

given up to transformations

$$
\left(\begin{array}{c}
\omega_{0}^{1} \\
\omega_{0}^{2} \\
\omega_{0}^{3} \\
\omega_{0}^{4}
\end{array}\right) \longmapsto\left(\begin{array}{cccc}
\mathrm{u}_{1} & 0 & 0 & 0 \\
\mathrm{u}_{2} & \mathrm{u}_{3} & 0 & 0 \\
\mathrm{u}_{4} & 0 & \mathrm{u}_{5} & \mathrm{u}_{6} \\
\mathrm{u}_{7} & 0 & \mathrm{u}_{8} & \mathrm{u}_{9}
\end{array}\right)\left(\begin{array}{c}
\omega_{0}^{1} \\
\omega_{0}^{2} \\
\omega_{0}^{3} \\
\omega_{0}^{4}
\end{array}\right)
$$

Within this coframe $\left\{\omega_{0}^{1}, \omega_{0}^{2}, \omega_{0}^{3}, \omega_{0}^{4}\right\}$, in terms of the two operators

$$
D:=\partial_{x}+p \partial_{z} \quad \text { and } \quad \Delta:=\partial_{y}+F \partial_{z}
$$

the exterior differential of any function $F=F(x, y, z, p)$ can be rewritten as

$$
\mathrm{d} F=F_{z} \omega_{0}^{1}+F_{p} \omega_{0}^{2}+D F \omega_{0}^{3}+\Delta F \omega_{0}^{4} .
$$

Proposition 5.1. The coframe of 1-forms $\left\{\omega_{0}^{1}, \omega_{0}^{2}, \omega_{0}^{3}, \omega_{0}^{4}\right\}$ modulo transformations (5.1) defines a para-CR structure of type $(1,1,2)$ if and only if

$$
0 \equiv D F=F_{x}+p F_{z}
$$

Proof. The only nontrivial integrability condition required to constitute a true para-CR structure comes from

$$
0=\mathrm{d} \omega_{0}^{1} \wedge \omega_{0}^{1} \wedge \omega_{0}^{2}=-D F \omega_{0}^{1} \wedge \omega_{0}^{2} \wedge \omega_{0}^{3} \wedge \omega_{0}^{4} .
$$

We will now show that for this class of para-CR structures there is an amazing coincidence between its main invariant, which will happen to be the Monge invariant with respect to $p$, and the classical Wünschmann invariant of the corresponding class of $3^{\text {rd }}$ order ODEs modulo point transformations.

From now on, we will only consider PDEs $z_{y}=F\left(x, y, z, z_{x}\right)$ satisfying $D F \equiv 0$. Furthermore, we will also assume that another point-invariant condition holds

$$
0 \neq F_{p p} \quad \text { (everywhere) }
$$

Cartan's process leads one to choose more convenient representatives of these forms

$$
\begin{aligned}
\omega^{1} & :=\omega_{0}^{1}, \\
\omega^{2} & :=\omega_{0}^{2}-\frac{\Delta F_{p p p} F_{p p}-\Delta F_{p p} F_{p p p}+3 F_{p} F_{p p} F_{z p p}-3 F_{p p}^{2} F_{z p}-2 F_{p} F_{p p p} F_{z p}}{6 F_{p p}^{3}} \omega_{0}^{1}, \\
\omega^{3} & :=\omega_{0}^{3}+F_{p} \omega_{0}^{4}-\frac{1}{3} \frac{F_{p p p}}{F_{p p}} \omega_{0}^{1}, \\
\omega^{4} & :=F_{p p} \omega_{0}^{4}+\frac{4 F_{p p p}^{2}-3 F_{p p} F_{p p p p}}{18 F_{p p}^{2}} \omega_{0}^{1},
\end{aligned}
$$

and we will use this choice in the sequel.

Using Cartan's method, it is then straightforward to solve the equivalence problem for point equivalence classes of such PDEs $z_{y}=F\left(x, y, z, z_{x}\right)$. The solution is summarized in the following 
Theorem 5.2. A PDE system $z_{y}=F\left(x, y, z, z_{x}\right)$ satisfying the two point-invariant conditions

$$
D F \equiv 0 \neq F_{z_{x} z_{x}},
$$

with its associated 1-forms $\omega^{1}, \omega^{2}, \omega^{3}, \omega^{4}$ as above, uniquely defines a 7-dimensional principal $H_{3}$-bundle $H_{3} \longrightarrow P_{7} \longrightarrow J_{4}$ over the space of first jets $J_{4} \ni(x, y, z, p)$ with the (reduced) structure group $\mathrm{H}_{3}$ consisting of matrices

$$
\left(\begin{array}{cccc}
\mathrm{u}_{3} \mathrm{u}_{5} & 0 & 0 & 0 \\
0 & \mathrm{u}_{3} & 0 & 0 \\
-\mathrm{u}_{3} \mathrm{u}_{8} & 0 & \mathrm{u}_{5} & 0 \\
-\frac{\mathrm{u}_{3} \mathrm{u}_{8}^{2}}{2 \mathrm{u}_{5}} & 0 & \mathrm{u}_{8} & \frac{\mathrm{u}_{5}}{\mathrm{u}_{3}}
\end{array}\right), \quad \mathrm{u}_{3} \in \mathbb{R}^{*}, \quad \mathrm{u}_{5} \in \mathbb{R}^{*}, \quad \mathrm{u}_{8} \in \mathbb{R}
$$

together with a unique coframe $\left\{\theta^{1}, \theta^{2}, \theta^{3}, \theta^{4}, \Omega_{1}, \Omega_{2}, \Omega_{3}\right\}$ on $P_{7}$ where

$$
\left(\begin{array}{l}
\theta^{1} \\
\theta^{2} \\
\theta^{3} \\
\theta^{4}
\end{array}\right):=\left(\begin{array}{cccc}
\mathrm{u}_{3} \mathrm{u}_{5} & 0 & 0 & 0 \\
0 & \mathrm{u}_{3} & 0 & 0 \\
-\mathrm{u}_{3} \mathrm{u}_{8} & 0 & \mathrm{u}_{5} & 0 \\
-\frac{\mathrm{u}_{3} \mathrm{u}_{8}^{2}}{2 \mathrm{u}_{5}} & 0 & \mathrm{u}_{8} & \frac{\mathrm{u}_{5}}{\mathrm{u}_{3}}
\end{array}\right)\left(\begin{array}{c}
\omega^{1} \\
\omega^{2} \\
\omega^{3} \\
\omega^{4}
\end{array}\right)
$$

such that the coframe enjoys precisely the structure equations (4.4). This time however, the curvature invariants $A_{1}, A_{2}, A_{3}, B_{1}, B_{2}, B_{3}, B_{4}, C_{1}, C_{2}, C_{3}, E_{1}, E_{2}$ depend on $F=F(x, y, z, p)$ and its derivatives up to order 6 .

Two relevant explicit expressions are

$$
A_{1}=-\frac{1}{54} \frac{1}{\mathrm{u}_{3}^{3}} \frac{M}{F_{p p}^{3}}, \quad C_{1}=\frac{1}{3} \frac{1}{\mathrm{u}_{3}^{2} \mathrm{u}_{5}} \frac{K}{F_{p p}^{5}},
$$

where

$$
\begin{aligned}
M:= & 9 F_{p p p p p} F_{p p}^{2}-45 F_{p p p p} F_{p p p} F_{p p}+40 F_{p p p}^{3} \\
K:= & \Delta F_{p p p p p} F_{p p}^{3}-5 \Delta F_{p p p p} F_{p p}^{2} F_{p p p}+12 \Delta F_{p p p} F_{p p} F_{p p p}^{2}-12 \Delta F_{p p} F_{p p p}^{3}-4 \Delta F_{p p p} F_{p p}^{2} F_{p p p p} \\
& +9 \Delta F_{p p} F_{p p} F_{p p p} F_{p p p p}-\Delta F_{p p} F_{p p}^{2} F_{p p p p p}+5 F_{p} F_{p p}^{3} F_{p p p p z}+6 F_{p p}^{4} F_{p p p z} \\
& -20 F_{p} F_{p p}^{2} F_{p p p} F_{p p p z}-12 F_{p p}^{3} F_{p p p} F_{p p z}+36 F_{p} F_{p p} F_{p p p}^{2} F_{p p z}-12 F_{p} F_{p p}^{2} F_{p p p p} F_{p p z} \\
& +8 F_{p p}^{2} F_{p p p}^{2} F_{p z}-24 F_{p} F_{p p p}^{3} F_{p z}-3 F_{p p}^{3} F_{p p p p} F_{p z}+18 F_{p} F_{p p} F_{p p p} F_{p p p p} F_{p z} \\
& -2 F_{p} F_{p p}^{2} F_{p p p p p} F_{p z} .
\end{aligned}
$$

Two equations $z_{y}=F\left(x, y, z, z_{x}\right)$ and $\bar{z}_{\bar{y}}=\bar{F}\left(\bar{x}, \bar{y}, \bar{z}, \bar{z}_{\bar{x}}\right)$ satisfying $D F=0 \neq F_{z_{x} z_{x}}$ and

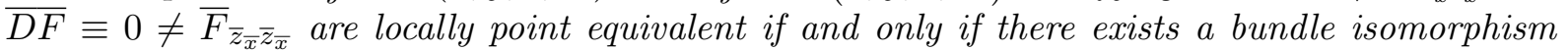
$\Phi: P_{7} \stackrel{\sim}{\longrightarrow} \bar{P}_{7}$ between the corresponding principal bundles $H_{3} \longrightarrow P_{7} \longrightarrow J_{4}$ and $\bar{H}_{3} \longrightarrow \bar{P}_{7} \longrightarrow$ $\bar{J}_{4}$ satisfying

$$
\Phi^{*} \bar{\theta}^{\mu}=\theta^{\mu} \quad \text { and } \quad \Phi^{*} \bar{\Omega}_{i}=\Omega_{i}, \quad \mu=1,2,3,4, \quad i=1,2,3 .
$$

This theorem enables one to think about the geometry of a PDE $z_{y}=F\left(x, y, z, z_{x}\right)$ with $D F \equiv 0 \neq F_{z_{x} z_{x}}$, considered modulo point transformations of variables, as the geometry of a certain $3^{\text {rd }}$ order ODE $y^{\prime \prime \prime}=H\left(x, y, y^{\prime}, y^{\prime \prime}\right)$, also considered modulo point transformations. In particular, one can ask how big is the subclass of point nonequivalent $3^{\text {rd }}$ order ODEs which are related to PDEs $z_{y}=F\left(x, y, z, z_{x}\right)$ with $D F \equiv 0 \neq F_{z_{x} z_{x}}$. 
We will not answer this question in this paper. Instead, we concentrate on the Einstein-Weyl geometric aspect of the above observation.

Since the EDS staying behind the PDEs $z_{y}=F\left(x, y, z, z_{x}\right)$ with $D F \equiv 0 \neq F_{z_{x} z_{x}}$ is visibly the same as the EDS for $3^{\text {rd }}$ order ODEs $y^{\prime \prime \prime}=H\left(x, y, y^{\prime}, y^{\prime \prime}\right)$, one can look for PDEs $z_{y}=F\left(x, y, z, z_{x}\right)$ with $D F \equiv 0 \neq F_{z_{x}} z_{x}$, which in addition satisfy $A_{1}=C_{1}=0$, and build a corresponding Einstein Weyl geometry, not in terms of $H\left(x, y, y^{\prime}, y^{\prime \prime}\right)$ satisfying $W(H) \equiv$ $C(H) \equiv 0$, but in terms of the function $F\left(x, y, z, z_{x}\right)$ satisfying $D F \equiv M(F) \equiv K(F) \equiv 0$. If only $\boldsymbol{M}(F) \equiv 0$, there exists a conformal Lorentzian metric on the leaf space of the integrable distribution in $P_{7}$ annihilated by $\left\{\theta^{1}, \theta^{3}, \theta^{4}\right\}$, and when moreover $K(F) \equiv 0$, all this produces Einstein-Weyl geometries. Actually, we gain the following

Theorem 5.3. A PDE $z_{y}=F\left(x, y, z, z_{x}\right)$ with $D F \equiv 0 \neq F_{z_{x} z_{x}}$ defines a bilinear form $\widetilde{g}$ of signature $(+,+,-, 0,0,0,0)$ on the bundle $P_{7} \ni\left(x, y, z, p, u_{3}, u_{5}, u_{8}\right)$ :

$$
\begin{aligned}
\widetilde{g}= & \theta^{3} \theta^{3}+\theta^{1} \theta^{4}+\theta^{4} \theta^{1}=\frac{\mathrm{u}_{5}^{2}}{9 F_{p p}^{2}}\left\{\left(3 F_{p p}\left[\mathrm{~d} x+F_{p} \mathrm{~d} y\right]-F_{p p p}[\mathrm{~d} z-p \mathrm{~d} x-F \mathrm{~d} y]\right)^{2}\right. \\
& \left.+(\mathrm{d} z-p \mathrm{~d} x-F \mathrm{~d} y)\left(18 F_{p p}^{3} \mathrm{~d} y+\left[4 F_{p p p}^{2}-3 F_{p p} F_{p p p p}\right][\mathrm{d} z-p \mathrm{~d} x-F \mathrm{~d} y]\right)\right\},
\end{aligned}
$$

degenerate along the rank 4 integrable distribution $\mathcal{D}_{4}$ which is the annihilator of $\theta^{1}, \theta^{3}, \theta^{4}$.

The PDE $z_{y}=F\left(x, y, z, z_{x}\right)$ with $D F \equiv 0 \neq F_{z_{x} z_{x}}$ also defines the 1-form

$$
\Omega_{3}:=r_{x} \mathrm{~d} x+r_{y} \mathrm{~d} y+r_{z} \mathrm{~d} z+\frac{1}{3} \mathrm{~d}\left[\log \left(u_{5}^{3} F_{p p}\right)\right],
$$

where

$$
\begin{aligned}
r_{x}= & \frac{1}{3 F_{p p}^{4}}\left\{\Delta F_{p p p} F_{p p}^{2}-\Delta F_{p p} F_{p p} F_{p p p}+3 F_{p} F_{p p}^{2} F_{p p z}-F_{p p}^{3} F_{p z}-2 F_{p} F_{p p} F_{p p p} F_{p z}\right. \\
& -\Delta F_{p p p p} F_{p p}^{2} p+3 \Delta F_{p p p} F_{p p} F_{p p p} p-3 \Delta F_{p p} F_{p p p}^{2} p+\Delta F_{p p} F_{p p} F_{p p p p} p \\
& -4 F_{p} F_{p p}^{2} F_{p p p z} p-2 F_{p p}^{3} F_{p p z} p+9 F_{p} F_{p p} F_{p p p} F_{p p z} p+F_{p p}^{2} F_{p p p} F_{p z} p \\
& \left.-6 F_{p} F_{p p p}^{2} F_{p z} p+2 F_{p} F_{p p} F_{p p p p} F_{p z} p\right\} \\
r_{y}= & \frac{1}{3 F_{p p}^{4}}\left\{-\Delta F_{p p p p} F F_{p p}^{2}+\Delta F_{p p p} F_{p} F_{p p}^{2}-\Delta F_{p p} F_{p p}^{3}+3 \Delta F_{p p p} F F_{p p} F_{p p p}\right. \\
& -\Delta F_{p p} F_{p} F_{p p} F_{p p p}-3 \Delta F_{p p} F F_{p p p}^{2}+\Delta F_{p p} F F_{p p} F_{p p p p}-4 F F_{p} F_{p p}^{2} F_{p p p z} \\
& +3 F_{p}^{2} F_{p p}^{2} F_{p p z}-2 F F_{p p}^{3} F_{p p z}+9 F F_{p} F_{p p} F_{p p p} F_{p p z}-3 F_{p} F_{p p}^{3} F_{p z} \\
& \left.-2 F_{p}^{2} F_{p p} F_{p p p} F_{p z}+F F_{p p}^{2} F_{p p p} F_{p z}-6 F F_{p} F_{p p p}^{2} F_{p z}+2 F F_{p} F_{p p} F_{p p p p} F_{p z}+3 F_{p p}^{4} F_{z}\right\}, \\
r_{z}= & \frac{1}{3 F_{p p}^{4}}\left\{\Delta F_{p p p p} F_{p p}^{2}-3 \Delta F_{p p p} F_{p p} F_{p p p}+3 \Delta F_{p p} F_{p p p}^{2}-\Delta F_{p p} F_{p p} F_{p p p p}+4 F_{p} F_{p p}^{2} F_{p p p z}\right. \\
& \left.+2 F_{p p}^{3} F_{p p z}-9 F_{p} F_{p p} F_{p p p} F_{p p z}-F_{p p}^{2} F_{p p p} F_{p z}+6 F_{p} F_{p p p}^{2} F_{p z}-2 F_{p} F_{p p} F_{p p p p} F_{p z}\right\} .
\end{aligned}
$$

The degenerate bilinear form $\tilde{g}$ descends to a Lorentzian conformal class $[g]$ on the leaf space $M_{3}$ of the distribution $\mathcal{D}_{4}$, if and only if the Monge invariant $\boldsymbol{M}(F) \equiv 0$ vanishes identically.

When $\boldsymbol{M}(F) \equiv 0$, the local coordinates on $M_{3}$ are $(x, y, z)$ with the projection

$$
\begin{aligned}
P_{7} & \longrightarrow M_{3}, \\
\left(x, y, z, p, \mathrm{u}_{3}, \mathrm{u}_{5}, \mathrm{u}_{8}\right) & \longmapsto(x, y, z),
\end{aligned}
$$

and the conformal class $[g]$ has a representative which is explicitly expressed in terms of $\mathrm{d} x, \mathrm{~d} y$, $\mathrm{d} z$, with coefficients depending only on $(x, y, z)$. 
Next, $\Omega_{3}$ descends to a 1-form denoted $A$ given up to the differential of a function on $M_{3} \ni$ $(x, y, z)$, if and only if $K(F) \equiv 0$.

Moreover, the pair $\left(\widetilde{g}, \Omega_{3}\right)$ descends to a representative of a Weyl structure $[(g, A)]$ on $M_{3}$, if and only if both $\boldsymbol{M}(F) \equiv 0$ and $K(F) \equiv 0$.

Finally, this Weyl structure is actually Einstein-Weyl, namely it satisfies (2.1).

\section{Transformation of the Wünschmann invariant into the Monge invariant}

As we now know, PDEs $z_{y}=F\left(x, y, z, z_{x}\right)$ with $D F \equiv 0 \neq F_{z_{x} z_{x}}$ satisfying $A_{1} \equiv 0 \equiv C_{1}$ always define an Einstein-Weyl geometry on the leaf space $M_{3}$ of the integrable distribution in $P_{7}$ annihilated by $\left\{\theta^{1}, \theta^{3}, \theta^{4}\right\}$.

The advantage of looking at a Weyl geometry from the $\mathrm{PDE} z_{y}=F\left(x, y, z, z_{x}\right)$ point of view rather than from the ODE side $y^{\prime \prime \prime}=H\left(x, y, y^{\prime}, y^{\prime \prime}\right)$, is that now the Wünschmann invariant of the ODE becomes the much simpler and classical Monge invariant

$$
A_{1}(H) \sim M(F)=9 F_{p p}^{2} F_{p p p p p}-45 F_{p p} F_{p p p p} F_{p p p}+40 F_{p p p}^{3} .
$$

Serendipitously, the identical vanishing $M(F) \equiv 0$ is well known to be equivalent to the condition that the graph of $p \longmapsto F(p)$ is contained in a conic of the $(p, F)$-plane, with parameters $(x, y, z)$. More precisely,

$$
0 \equiv M(F) \quad \Longleftrightarrow \quad \mathrm{A} F^{2}+2 \mathrm{~B} F p+\mathrm{C} p^{2}+2 \mathrm{~K} F+2 \mathrm{~L} p+\mathrm{M} \equiv 0,
$$

for some functions $\mathrm{A}, \mathrm{B}, \mathrm{C}, \mathrm{K}, \mathrm{L}, \mathrm{M}$ depending only on $(x, y, z)$.

Thus, passing from the formulation of Einstein-Weyl's equations in terms of a $3^{\text {rd }}$ order ODE $y^{\prime \prime \prime}=H\left(x, y, y^{\prime}, y^{\prime \prime}\right)$ to the formulation in terms of a $\operatorname{PDE} z_{y}=F\left(x, y, z, z_{x}\right)$, we are able to find a rather large class of solutions to the equation

$$
W(H) \equiv 0 .
$$

Indeed, by replacing $W(H) \sim M(F)$, the solution (6.1) is just conical!

\section{How to construct new explicit Lorentzian Einstein-Weyl metrics?}

But remember we also have to assure that

$$
0 \equiv D F=\partial_{x} F+p \partial_{z} F .
$$

The simultaneous conditions $D F \equiv 0 \equiv M(F)$ can be achieved for instance by taking $F$ satisfying

$$
a F^{2}+2 b F(z-p x)+c(z-p x)^{2}+2 k F+2 l(z-p x)+m \equiv 0,
$$

with $a, b, c, k, l, m$ being now functions of $y$ only!

From now on, we will analyze this special solution for $M(F) \equiv 0 \equiv D F$. The simplest case occurs when avoiding square root by choosing

$$
a:=0,
$$

so that

$$
F:=\frac{-c(z-x p)^{2}-2 l(z-x p)-m}{2 b(z-x p)+2 k} .
$$


Here

$$
b=b(y), \quad c=c(y), \quad k=k(y), \quad l=l(y), \quad m=m(y)
$$

are free arbitrary differentiable functions of one variable $y$.

A direct check shows that remarkably this solution (7.1) also satisfies $K(F) \equiv 0$ !

Proposition 7.1. All such

$$
F:=\frac{-c(z-x p)^{2}-2 l(z-x p)-m}{2 b(z-x p)+2 k}
$$

with any functions $b, c, k, l, m$ of $y$, lead to Einstein-Weyl structures in 3-dimensions.

Performing the Cartan procedure to determine the coframe $\left\{\theta^{1}, \theta^{2}, \theta^{3}, \theta^{4}, \Omega_{1}, \Omega_{2}, \Omega_{3}\right\}$, projecting both $\theta^{3} \theta^{3}+\theta^{1} \theta^{4}+\theta^{4} \theta^{1}$ and $\Omega_{3}$ to the leaf space of the annihilator $M^{3}$ of $\left\{\theta^{1}, \theta^{3}, \theta^{4}\right\}$, equipping $M_{3} \equiv \mathbb{R}^{3}$ with coordinates $(x, y, z)$, we therefore obtain functionally parameterized Einstein-Weyl structures $(g, A)$ on $\mathbb{R}^{3} \ni(x, y, z)$ represented by the signature $(2,1)$ Lorentzian metric

$$
\begin{aligned}
g:= & (k+b z)^{2} \mathrm{~d} x^{2}+x^{2}\left(l^{2}-c m\right) \mathrm{d} y^{2}+x^{2} b^{2} \mathrm{~d} z^{2} \\
& +2 x(c k z-b l z+k l-b m) \mathrm{d} x \mathrm{~d} y-2 x \boldsymbol{b}(\boldsymbol{k}+\boldsymbol{b} z) \mathrm{d} x \mathrm{~d} z-2 x^{2}(c k-b l) \mathrm{d} y \mathrm{~d} z,
\end{aligned}
$$

together with the differential 1-form

$$
\begin{aligned}
A:= & \frac{-c k+b l+b^{\prime} k-b k^{\prime}}{x\left(c k^{2}-2 b k l+b^{2} m\right)}(x b \mathrm{~d} z-(k+b z) \mathrm{d} x) \\
& +\frac{b l^{2}-c b m-b^{\prime} k l+b b^{\prime} m+c k k^{\prime}-b k^{\prime} l}{c k^{2}-2 b k l+b^{2} m} \mathrm{~d} y .
\end{aligned}
$$

An independent direct check confirms that equations (1.1) are indeed identically fulfilled.

As regards the Cotton tensor, we compute its 5 components, and find that they are not identically zero. Hence the obtained Einstein-Weyl structures are generically conformally nonflat. Thus, Theorem 1.1 is established. The story for Theorem 1.4 is quite similar.

Next, without assuming $\mathrm{A} \equiv 0$ in (6.1), let us now make the ansatz that

$$
a F^{2}+2 b F(z-x p)+c(z-x p)^{2}+2 k F+2 l(z-x p)+m \equiv 0,
$$

for some arbitrary functions $a, b, c, k, l, m$ of $y$. The (two) solutions $F$ automatically satisfy $D F \equiv 0 \equiv M(F)$.

Since the solutions to Monge's equation are conics in the $(p, F)$-plane, we can rewrite in a hyperbolic setting

$$
(a F+b(z-x p)+c)^{2}-(k F+l(z-x p)+m)^{2} \equiv 1,
$$

with changed functions $a, b, c, k, l, m$ of $y$. To avoid transcendental functions in computations, we parametrize $\cosh t=\frac{1+q^{2}}{2 q}$ and $\sinh t=\frac{1-q^{2}}{2 q}$, and then, solving for $F$ and for $z-x p$, we may start from

$$
\begin{aligned}
& F=a(y) \frac{1+q^{2}}{2 q}+b(y) \frac{1-q^{2}}{2 q}+c(y), \\
& z-x p=k(y) \frac{1+q^{2}}{2 q}+l(y) \frac{1-q^{2}}{2 q}+m(y),
\end{aligned}
$$

again with (changed) free functions $a, b, c, k, l, m$ of $y$. Taking

$$
\omega_{0}^{1}:=\mathrm{d}(z-x p)+x \mathrm{~d} p-F \mathrm{~d} y, \quad \omega_{0}^{2}:=\mathrm{d} x, \quad \omega_{0}^{3}:=\mathrm{d} y, \quad \omega_{0}^{4}:=\mathrm{d} p,
$$

and performing para-CR Cartan reduction to an $\{e\}$-structure/connection, we obtain 
Proposition 7.2. The second invariant condition $K(F) \equiv 0$ holds precisely in the following two cases:

(1) $k=l$;

(2) $c=m^{\prime}$ and $a=\frac{b l+k k^{\prime}-l l^{\prime}}{k}$. by

In case (1), we obtain Einstein-Weyl structures for all free functions $a, b, c, l, m$ of $y$ given

$$
g:=2 \tau^{1} \tau^{2}+\left(\tau^{3}\right)^{2}, \quad A:=-\frac{2(a+b)}{x(a-b) l} \tau^{2}-\frac{c-m^{\prime}}{x(a-b)} \tau^{3},
$$

where

$$
\tau^{1}:=x(\boldsymbol{a}+\boldsymbol{b}) \mathrm{d} y-2 l \mathrm{~d} x, \quad \tau^{2}:=-\frac{1}{2} x(\boldsymbol{a}-\boldsymbol{b}) \mathrm{d} y, \quad \tau^{3}:=x \boldsymbol{c} \mathrm{d} y-x \mathrm{~d} z+(z-m) \mathrm{d} x .
$$

We verify that these Einstein-Weyl structures have nontrivial $F=\mathrm{d} A \not \equiv 0$ and nontrivial Cotton $([g]) \not \equiv 0$.

In case (2), we obtain Einstein-Weyl structures given by

$$
g:=2 \tau^{1} \tau^{2}+\left(\tau^{3}\right)^{2}, \quad A:=\mathrm{d}\left[\log \left(x^{2} e\right)\right],
$$

where

$$
\begin{aligned}
\tau^{1} & :=(k+l) k \mathrm{~d} x+x\left(b k-b l+k k^{\prime}-l l^{\prime}\right) \mathrm{d} y, \\
\tau^{2} & :=\frac{1}{2}(k-l) k \mathrm{~d} x+\frac{1}{2} x\left(b k-b l+k k^{\prime}-l l^{\prime}\right) \mathrm{d} y, \\
\tau^{3} & :=-(z-m) k \mathrm{~d} x-x k m \mathrm{~d} y+x k \mathrm{~d} z .
\end{aligned}
$$

But this structure, which depends on 3 functions $b, k, l$ of $y$, is flat

$$
\mathrm{d} A \equiv 0 \equiv \operatorname{Cotton}([g]) .
$$

Finally, without replacing $p$ by $z-x p$, let us make the ansatz that

$$
a F^{2}+2 b F p+c p^{2}+2 k F+2 l p+m \equiv 0 .
$$

Dealing similarly with the hyperbolic case,

$$
\begin{aligned}
& F=a(y) \frac{1+q^{2}}{2 q}+b(y) \frac{1-q^{2}}{2 q}+c(y), \\
& p=k(y) \frac{1+q^{2}}{2 q}+l(y) \frac{1-q^{2}}{2 q}+m(y),
\end{aligned}
$$

we obtain nontrivial Einstein-Weyl structures. For instance, when $k=l$ as in (1) above

$$
g:=2 \tau^{1} \tau^{2}+\left(\tau^{3}\right)^{2}, \quad A:=-\frac{m^{\prime}}{(a-b) l} \tau^{3},
$$

where

$$
\tau^{1}:=2 l \mathrm{~d} x+(\boldsymbol{a}+\boldsymbol{b}) \mathrm{d} y, \quad \tau^{2}:=-\frac{1}{2}(\boldsymbol{a}-\boldsymbol{b}) \mathrm{d} y, \quad \tau^{3}:=\mathrm{d} z-m \mathrm{~d} x+(\boldsymbol{a}+\boldsymbol{b}) \mathrm{d} y .
$$

Note that this is again nontrivial

$$
\mathrm{d} A \not \equiv 0 \not \equiv \operatorname{Cotton}([g]) \text {. }
$$

and note that we do not have $x, z$ dependence here. 


\section{Transforming $z_{y}=F\left(z_{x}\right)$ into $w^{\prime \prime \prime}=w^{\prime \prime} H(t)$}

We end up by exploring a link between our PDE systems and $3^{\text {rd }}$ order ODEs. For simplicity, we will assume that $F=F\left(z_{x}\right)$ depends only on $p=z_{x}$.

To avoid notational confusion, $3^{\text {rd }}$-order ODEs will now be denoted as $w^{\prime \prime \prime}=H\left(t, w, w^{\prime}, w^{\prime \prime}\right)$, and the fundamental 1-forms as

$$
\begin{array}{ll}
\omega^{1}:=\mathrm{d} z-p \mathrm{~d} x-F(p) \mathrm{d} y, & \theta^{1}:=\mathrm{d} w-w_{1} \mathrm{~d} t, \\
\omega^{2}:=\mathrm{d} p, & \theta^{2}:=\mathrm{d} t, \\
\omega^{3}:=\mathrm{d} x, & \theta^{3}:=\mathrm{d} w_{1}-w_{2} \mathrm{~d} t, \\
\omega^{4}:=\mathrm{d} y, & \theta^{4}:=\mathrm{d} w_{2}-H\left(t, w, w_{1}, w_{2}\right) \mathrm{d} t .
\end{array}
$$

We ask what equivalence class of $3^{\text {rd }}$-order ODE's corresponds to the equivalence class of PDEs $z_{y}=F\left(z_{x}\right)$, still with $F_{p p} \neq 0$, and under the $G$-structures of Sections 4 and 5 .

For this, since $\omega^{1}$ and $\theta^{1}$ are both defined up to plain dilations $\omega^{1} \sim \mathrm{u} \omega^{1}$ and $\theta^{1} \sim \mathrm{u} \theta^{1}$, we transform $\omega^{1}$ in order to make the shape of $\theta^{1}$ appear, using that $F$ depends only on $p$

$$
\omega^{1}=\mathrm{d}(z-x p-y F(p))-\left(-x-y F_{p}(p)\right) \mathrm{d} p=: \mathrm{d} w-w_{1} \mathrm{~d} t
$$

with $t:=p, w:=z-x p-y F(p), w_{1}:=-x-y F_{p}(p)$. With this, $\omega^{2}=\mathrm{d} p=\mathrm{d} t=\theta^{2}$. Next, using $\omega^{3} \sim-\omega^{3}-\underline{\mathrm{ud} y}$, it comes

$$
\begin{aligned}
\omega^{3}=\mathrm{d} x & =-\left[\mathrm{d}\left(-x-y F_{p}(p)\right)+y F_{p p}(p) \mathrm{d} p+F_{p}(p) \mathrm{d} y\right] \\
& \sim\left[\mathrm{d} w_{1}+y F_{p p}(p) \mathrm{d} p+F_{p}(p) \mathrm{d} y\right]-\underline{F_{p}(p) \mathrm{d} y}=\mathrm{d} w_{1}-\left(-y F_{p p}(p)\right) \mathrm{d} p,
\end{aligned}
$$

whence $w_{2}:=-y F_{p p}(p)$.

A last computation using $\omega^{4} \sim \mathrm{u} \omega^{4}$

$$
\omega^{4}=\mathrm{d} y=-\frac{1}{F_{p p}(p)}\left[\mathrm{d}\left(-y F_{p p}(p)\right)+y F_{p p p}(p) \mathrm{d} p\right] \sim \mathrm{d} w_{2}-\left(-y F_{p p p}(p) \mathrm{d} p\right),
$$

shows that the right-hand side function $H=H\left(t, w_{2}\right)$ of the associated ODE $w^{\prime \prime \prime}=H$ is independent of $w, w_{1}$ as it must be

$$
H:=-y F_{p p p}(p)=w_{2} \frac{F_{t t t}(t)}{F_{t t}(t)} .
$$

Hence

$$
w^{\prime \prime \prime}=w^{\prime \prime} \frac{F_{t t t}(t)}{F_{t t}(t)}
$$

is the $3^{\text {rd }}$ order ODE associated to the para-CR structure given by $z_{y}=F\left(z_{x}\right)$. Observe that $z_{y}=\frac{1}{2} z_{x}^{2}$ becomes $w^{\prime \prime \prime}=0$, leading to the flat Einstein-Weyl structure.

Assertion 8.1. The Wünschmann invariant for $O D E s w^{\prime \prime \prime}=w^{\prime \prime} \frac{F_{t t t}(t)}{F_{t t}(t)}$, where $F(t)$ with $F_{t t} \neq 0$ is an arbitrary function of one variable, corresponds to the Monge invariant of the PDE $z_{y}=$ $F\left(z_{x}\right)$ :

$$
\text { Wünschmann }\left(w_{2} \frac{F_{t t t}(t)}{F_{t t}(t)}\right)=\frac{9 F_{t t}(t) F_{t t t t t}(t)-45 F_{t t} F_{t t t} F_{t t t t}+40 F_{t t t}^{3}}{F_{t t}^{3}}=\frac{\operatorname{Monge}(F)}{F_{t t}^{3}} .
$$

Proof. Among the 25 terms of Wünschmann's invariant shown in the Introduction, only 7 remain thanks to $0 \equiv H_{w} \equiv H_{w_{1}}$ :

$$
\begin{aligned}
\text { Wünschmann }\left(H\left(t, w_{2}\right)\right)= & -9 H H_{w_{2}} H_{w_{2} w_{2}}-9 H_{t} H_{w_{2} w_{2}}+18 H H_{t w_{2} w_{2}}-18 H_{w_{2}} H_{t w_{2}} \\
& +9 H_{t t w_{2}}+4 H_{w_{2}}^{3}+9 H^{2} H_{w_{2} w_{2} w_{2}},
\end{aligned}
$$

and a direct substitution of $H:=w_{2} \frac{F_{t t t}(t)}{F_{t t}(t)}$ leads to the result. 


\section{Acknowledgements}

Insights of the anonymous referees are gratefully acknowledged. This collaboration is supported by the National Science Center, Poland, grant number 2018/29/B/ST1/02583.

\section{References}

[1] Calderbank D.M.J., Pedersen H., Einstein-Weyl geometry, in Surveys in Differential Geometry: Essays on Einstein Manifolds, Surv. Differ. Geom., Vol. 6, Int. Press, Boston, MA, 1999, 387-423.

[2] Cartan E., La geometria de las ecuaciones diferenciales de tercer orden, Rev. Mat. Hispano-Amer. 4 (1941), $1-31$.

[3] Cartan E., Sur une classe d'espaces de Weyl, Ann. Sci. École Norm. Sup. 60 (1943), 1-16.

[4] Dunajski M., Mason L.J., Tod P., Einstein-Weyl geometry, the dKP equation and twistor theory, J. Geom. Phys. 37 (2001), 63-93, arXiv:math.DG/0004031.

[5] Eastwood M.G., Tod K.P., Local constraints on Einstein-Weyl geometries: the 3-dimensional case, Ann. Global Anal. Geom. 18 (2000), 1-27.

[6] Frittelli S., Kozameh C., Newman E.T., Differential geometry from differential equations, Comm. Math. Phys. 223 (2001), 383-408, arXiv:gr-qc/0012058.

[7] Godlinski M., Geometry of third-order ordinary differential equations and its applications in general relativity, arXiv:0810.2234.

[8] Godlinski M., Nurowski P., Geometry of third order ODEs, arXiv:0902.4129.

[9] Hill C.D., Nurowski P., Differential equations and para-CR structures, Boll. Unione Mat. Ital. 3 (2010), 25-91, arXiv:0909.2458.

[10] Hitchin N.J., Complex manifolds and Einstein's equations, in Twistor Geometry and Nonlinear Systems (Primorsko, 1980), Lecture Notes in Math., Vol. 970, Springer, Berlin - New York, 1982, 73-99.

[11] Jones P.E., Tod K.P., Minitwistor spaces and Einstein-Weyl spaces, Classical Quantum Gravity 2 (1985), $565-577$.

[12] LeBrun C., Mason L.J., The Einstein-Weyl equations, scattering maps, and holomorphic disks, Math. Res. Lett. 16 (2009), 291-301, arXiv:0806.3761.

[13] Merker J., Lie symmetries and CR geometry, J. Math. Sci. 154 (2008), 817-922, arXiv:math.CV/0703130.

[14] Merker J., Nurowski P., On degenerate para-CR structures: Cartan reduction and homogeneous models, arXiv:2003.08166.

[15] Merker J., Pocchiola S., Explicit absolute parallelism for 2-nondegenerate real hypersurfaces $M^{5} \subset \mathbb{C}^{3}$ of constant Levi rank 1, J. Geom. Anal. 30 (2020), 2689-2730.

[16] Monge G., Sur les équations différentielles des courbes du second degré, Corr. l'École Impériale Polytech. (1810), no. 2, 51-54; Bull. Soc. Philomat. Paris (1810), 87-88.

[17] Nurowski P., Differential equations and conformal structures, J. Geom. Phys. 55 (2005), 19-49, arXiv:math.DG/0406400.

[18] Pedersen H., Tod K.P., Three-dimensional Einstein-Weyl geometry, Adv. Math. 97 (1993), 74-109.

[19] Tod K.P., Compact 3-dimensional Einstein-Weyl structures, J. London Math. Soc. 45 (1992), 341-351.

[20] Tod K.P., Einstein-Weyl spaces and third-order differential equations, J. Math. Phys. 41 (2000), 5572-5581.

[21] Weyl H., Raum, Zeit, Materie, Springer-Verlag, Berlin, 1919.

[22] Wünschmann K., Uber Berührungsbedingungen bei Integralkurven von Differentialgleichungen, Inaug. Dissert., Leipzig, Teubner, 1905. 